\title{
LETTER
}

\section{Causal interpretation requires appropriate study design}

\author{
P. C. Priest
}

Received: 9 March 2009 / Accepted: 11 March 2009 /Published online: 27 March 2009

(C) Springer-Verlag 2009

To the Editor: I read with interest the paper by Richardson et al. published in Diabetologia [1]. The paper reports a sort of case-control study, but gives very little information about the age or other demographic or clinical characteristics of the cases and controls, so it is not possible to properly assess the appropriateness of the control group. At the very least, it is unlikely to represent an unbiased sample of the population from which the cases arose (which is not described). For example, would one expect neonates to have had the opportunity to be infected with enterovirus? Presumably not. Therefore, except for neonatal cases (of which there were none), neonates should not be used as controls.

Depending on their cause of death and other factors, the paediatric controls may well not be representative of the source population in terms of their enterovirus infection experience. If this is the case (and it is not possible from the paper to evaluate this), the results of the study would be meaningless. Without more information about both cases and controls, they are uninterpretable.

Duality of interest The author declares that there is no duality of interest associated with this manuscript.

\section{Reference}

1. Richardson SJ, Willcox A, Bone AJ, Foulis AK, Morgan NG (2009) The prevalence of enteroviral capsid protein vp1 immunostaining in pancreatic islets in human type 1 diabetes. Diabetologia doi:10.1007/s00125-009-1276-0

P. C. Priest $(\bowtie)$

Department of Preventive and Social Medicine,

University of Otago,

P.O. Box 913, Dunedin 9054, New Zealand

e-mail: patricia.priest@otago.ac.nz 